\title{
How are family doctors serving the Hong Kong community during the COVID-19 outbreak? A survey of HKCFP members
}

\section{Esther YT Yu, Will LH Leung, Samuel YS Wong *, Kiki SN Liu, Eric YF Wan; for the HKCFP Executive and Research Committee}

\section{A B S T R A C T}

Introduction: This study evaluated the preparedness of family doctors during the early phase of the coronavirus disease 2019 (COVID-19) outbreak in Hong Kong.

Methods: All members of the Hong Kong College of Family Physicians were invited to participate in a cross-sectional online survey using a 20 -item questionnaire to collect information on practice preparedness for the COVID-19 outbreak through an email followed by a reminder SMS message between 31 January 2020 and 3 February 2020.

Results: Of 1589 family doctors invited, 491 (31\%) participated in the survey, including 242 (49\%) from private sector. In all, $98 \%$ surveyed doctors continued to provide clinical services during the survey period, but reduced clinic service demands were observed in $45 \%$ private practices and $24 \%$ public clinics. Almost all wore masks during consultation and washed hands between or before patient contact. Significantly more private than public doctors ( $80 \%$ vs $26 \%, \mathrm{P}<0.001$ ) experienced difficulties in stocking personal protective equipment (PPE); more public doctors used guidelines to manage suspected patients. The main concern of the respondents was

This article was published on 1 Jun 2020 at www.hkmj.org. PPE shortage. Respondents appealed for effective public health interventions including border control, quarantine measures, designated clinic setup, and public education.

Conclusion: Family doctors from public and private sectors demonstrated preparedness to serve the community from the early phase of the COVID-19 outbreak with heightened infection control measures and use of guidelines. However, there is a need for support from local health authorities to secure PPE supply and institute public health interventions.

\begin{tabular}{l} 
Hong Kong Med J 2020;26:176-83 \\
\hline https://doi.org/10.12809/hkmj208606 \\
${ }_{1,2}$ EYT Yu, MB, BS, FHKAM (Family Medicine) \\
${ }^{1,3}$ WLH Leung, FHKAM (Family Medicine), MScHSM (CUHK) \\
${ }^{1,4}$ SYS Wong *, MD, FHKAM (Family Medicine) \\
${ }^{2}$ KSN Liu, BSc \\
${ }^{2,5}$ EYF Wan, PhD \\
${ }^{1}$ The Hong Kong College of Family Physicians, Hong Kong \\
${ }^{2}$ Department of Family Medicine and Primary Care, The University of \\
Hong Kong, Hong Kong \\
${ }^{3}$ Department of Family Medicine and Primary Health Care, Kowloon West \\
Cluster, Hospital Authority, Hong Kong \\
${ }^{4}$ The Jockey Club School of Public Health and Primary Care, The Chinese \\
University of Hong Kong, Hong Kong \\
${ }^{5}$ Department of Pharmacology and Pharmacy, The University of Hong \\
Kong, Hong Kong \\
${ }^{*}$ Corresponding author: yeungshanwong@cuhk.edu.hk
\end{tabular}

New knowledge added by this study

- The coronavirus disease 2019 (COVID-19) outbreak in Hong Kong resulted in reduced primary care service demands and abrupt shortage of personal protective equipment (PPE) among primary care clinics.

- The majority of surveyed Hong Kong family doctors consistently adopted facemask wearing and handwashing for infection control at their practice.

- Public health measures including border control, quarantine, and public education were advocated as important interventions to limit the spread of COVID-19.

Implications for clinical practice or policy

- Family doctors in Hong Kong from both public and private sectors were willing and prepared to provide firstcontact clinical service to the community during the COVID-19 outbreak.

- Family doctors in Hong Kong needed better support from local health authorities on PPE supply, guided management of patients with COVID-19, greater availability of rapid diagnostic tests, and complementary public health interventions.

- Better coordination between public and private sectors is crucial, to include private family doctors as part of the overall health system strategy and emergency responses, because $70 \%$ of primary care consultations take place in the private sector in Hong Kong. 


\section{Introduction}

Family doctors, serving as the first point of professional contact for patients, are inevitably first to identify probable cases of coronavirus disease 2019 (COVID-19) among the many patients presenting with respiratory symptoms each day. ${ }^{1}$ Family doctors in Hong Kong have experience in dealing with the severe acute respiratory syndrome (SARS) epidemic in $2003^{2,3}$ and the H1N1 pandemic in 2009., However, their preparedness in handling another outbreak of a novel infectious disease has not been explored. Furthermore, Hong Kong has a dual-track healthcare system in which $70 \%$ of primary medical care, especially acute episodic care, is provided in the private sector where practice settings and resources vary and differ from those of public clinics. ${ }^{6-8}$ Family doctors play a crucial role in the community to offer first contact and coordinated care for patients, and their preparedness, perceptions, and attitudes towards COVID-19 are particularly important to inform future strategies for responding to epidemics. Hence, the Hong Kong College of Family Physicians (HKCFP) conducted an online survey among its members to evaluate preparedness and to identify clinic-related challenges of private and public family doctors who were providing primary care services during the early phase of the evolving COVID-19 outbreak in Hong Kong.

\section{Methods}

All family doctors who are HKCFP members were invited to complete an online survey. The structured questionnaire (online supplementary Appendix 1) comprised 20 questions. Twelve closed-ended questions assessed the effects of the COVID-19 outbreak (at the time of the survey, the World Health Organization had not announced it as a pandemic) on clinical services and the preparedness of the responding family doctors, such as changes in infection control practice. An open-ended question invited respondents to express their concerns towards the COVID-19 outbreak and suggest measures that would facilitate their clinical practice. The last seven questions collected demographics of the respondents. The survey questions were modified from a previous survey for primary care doctors in Hong Kong and Canada ${ }^{9,10}$ and pilot-tested by a panel of experienced academic family doctors and HKCFP Research and Executive Committee members. Invitation e-mails and short message service reminders were sent to target participants between 31 January 2020 and 3 February 2020.

Descriptive statistics were used to summarise the characteristics of the respondents. Respondents were stratified by their practice sector (ie, public vs private). The differences in the effects of the COVID-19 outbreak on the clinical practices, clinic

\section{香港家庭醫學學院會員問卷調查：2019冠狀病 毒病爆發期間家庭醫生在社區服務的情況 \\ 余㦤德、梁樂行、黃仰山、廖思雅、尹旭輝；香港家庭 醫學學院常務及研究委員會}

引言：本研究旨在評估香港2019冠狀病毒病（COVID-19）爆發早期 家庭醫生的準備情況。

方法: 邀請香港家庭醫學學院所有會員參加一項橫斷面在線調查, 問 卷共20項目，於2020年1月31日至2020年2月3日期間進行, 並通過 電子郵件和提醒短訊方式收集有關COVID-19爆發的實踐準備情況資 料。

結果：被邀請的1589名家庭醫生中, 491名（31\%）參與問卷調查， 其中242名（49\%）為私家醫生。問卷調查期間98\%受訪醫生繼續提供 臨床服務, 但 $45 \%$ 私家醫生和 $24 \%$ 公立醫院醫生表示服務需求則有所 減少。絕大部份受訪醫生表示問診時會戴口罩以及接觸患者之前或期 間會洗手。較多私家醫生表示個人防護裝備存量不足（ $80 \%$ 比 $26 \%$, $\mathrm{P}<0.001)$, 較多公立醫院醫生會使用相關指引管理可疑患者。受訪 者最關注的是個人防護裝備短缺問題。他們也呼㫻賞局應採取有效公 共衛生干預措施, 包括邊境管制、檢疫措施、指定診所設置和公眾教 育。

結論：公立醫院和私家家庭醫生在COVID-19爆發初期便作好為社區 服務的準備並加強感染控制措施和準則, 然而, 個人防護裝備供應和 採取公共衛生干預措施還需要有關當局支持。

service, and infection control practices between public and private family doctors were evaluated by Pearson Chi squared test. Thematic analysis was performed on the respondents' free comments and suggestions. The responses were reviewed independently by two investigators and consolidated into themes. Inconsistencies were resolved by discussion between the two investigators to reach consensus on a common theme. The consolidated themes from the respondents' suggestions and concerns were further stratified by respondents' practice sector using descriptive statistics.

All significance tests were two-tailed and those with a $P$ value of $<0.05$ were considered statistically significant. The statistical analysis was executed by Stata (Version 16.0; StataCorp LLC, College Station [TX], US).

Since the survey was initially conducted to examine the needs and preparedness of frontline family doctors who are members of the HKCFP during the early phase of COVID-19 outbreak in Hong Kong, ethics approval was obtained from the Survey and Behavioural Research Ethics Committee of The Chinese University of Hong Kong subsequently for data analysis and presentation.

The Strengthening the Reporting of Observational Studies in Epidemiology (STROBE) checklist for cross-sectional studies was used in the drafting of this article. ${ }^{11}$ 


\section{Results}

Of 1589 HKCFP members invited to complete the survey, $491(31 \%)$ provided a complete and valid response (Table 1). Of the respondents, 393 $(80 \%)$ had attained higher qualifications in Family Medicine. Among the respondents, 236 (48\%) worked at public primary care clinics operated by the Hospital Authority or Department of Health, and $242(49 \%)$ worked in the private sector, half of whom were solo practitioners. The ratio of public to private sector respondents was approximately 1:1.

\section{Effects of the COVID-19 outbreak on clinical practices and regular clinic services}

The vast majority of the respondents $(n=482,98 \%)$

TABLE I. Characteristics of respondents*

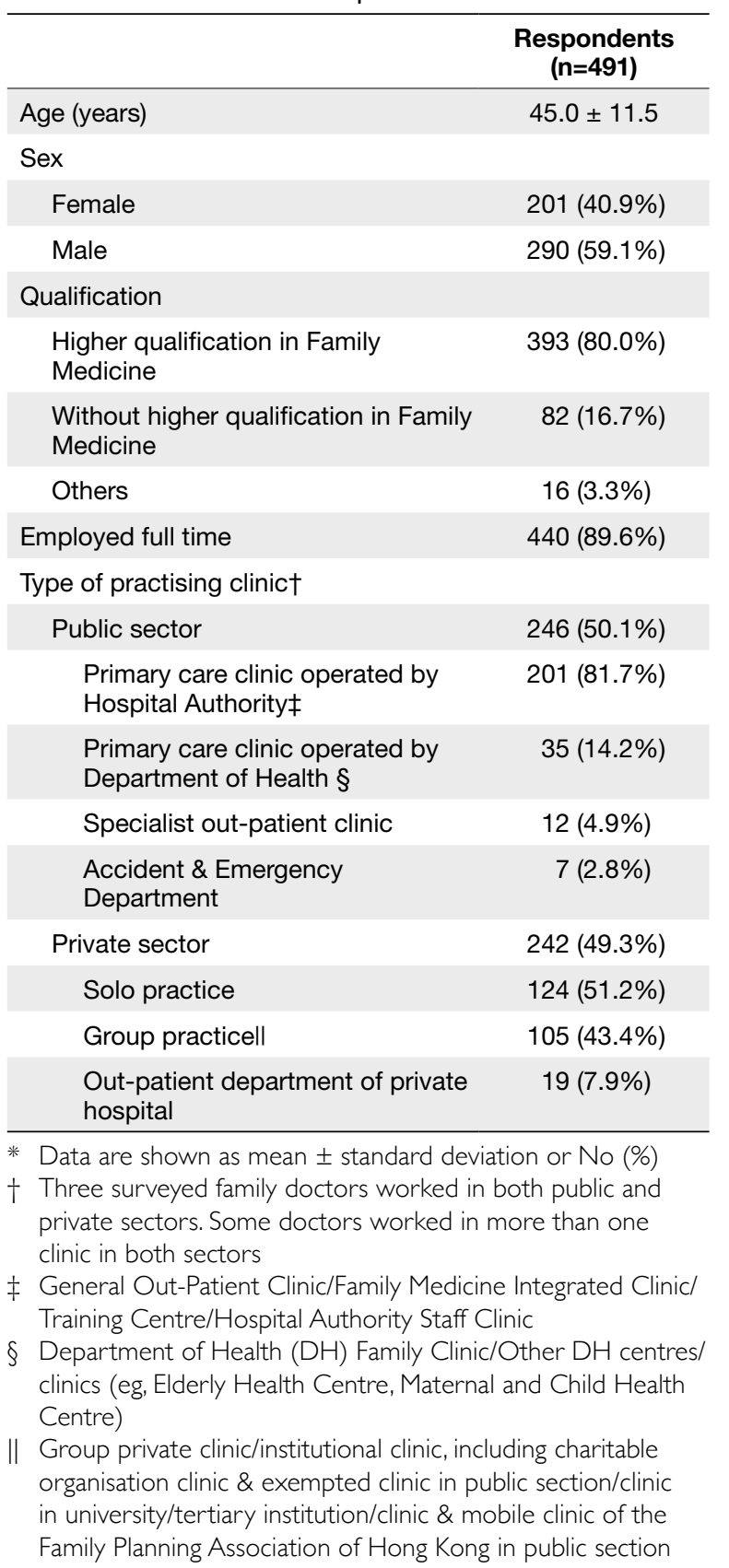

continued to provide clinic services although most of their clinic practices $(n=428,87 \%)$ had been affected by the COVID-19 outbreak (Fig 1, online supplementary Appendix 2). Significantly a higher proportion of private than public family doctors reported reduced clinic service demands during this outbreak ( $\mathrm{n}=111$ [45\%] vs $\mathrm{n}=60$ [24\%], $\mathrm{P}<0.001)$. Half of the surveyed family doctors adjusted non-acute

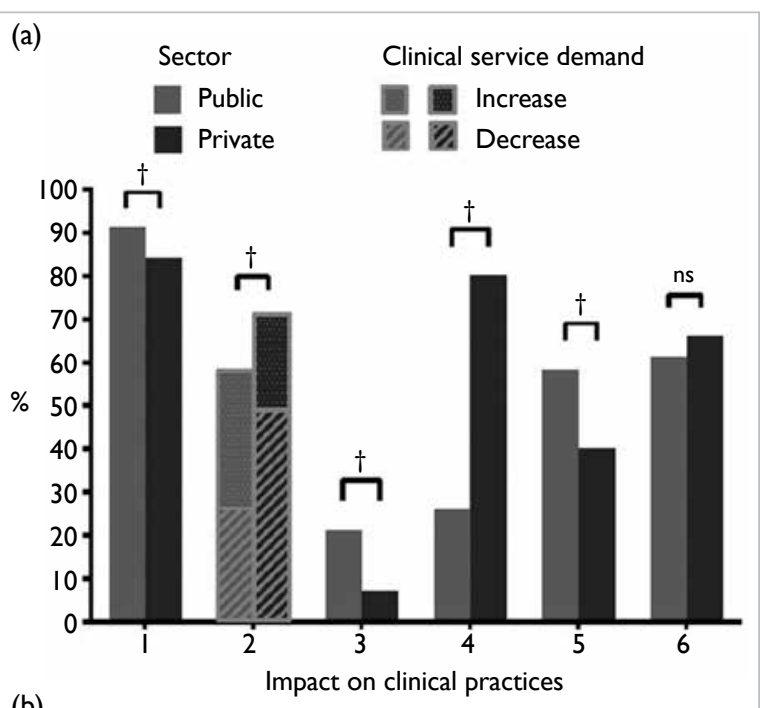

(b)

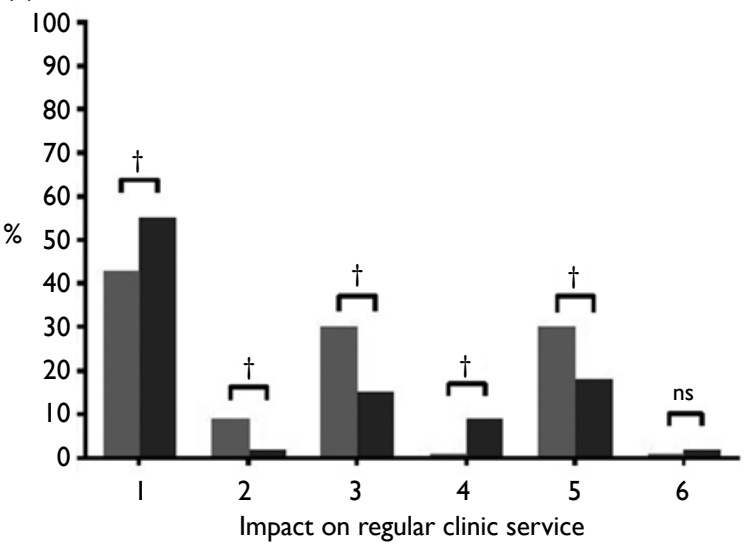

FIG I. Effects of the COVID-19 outbreak on (a) clinical practices and (b) regular clinic service of family doctors in the public and private sectors in Hong Kong*

Abbreviations: COVID-19 = coronavirus disease 2019; ns = not significant

* Impact on clinical practices: I = Clinical practice affected; $2=$ Change in service demands (increase or decrease); $3=$ Encounter patients with suspected/confirmed COVID-19; 4 = Problems in stocking personal protective equipment; 5 = Use of guideline to assist clinical decision for diagnosing COVID- 19 infection; 6 = Need for training to deal with the COVID-19 outbreak. Impact on regular clinic service: I = No change needed to sustain regular essential primary care services; 2 = Increase office hours; 3 Shorten patient consultation time; 4 = Ask patients to go to other clinics; 5 = Cancel or change regular non-acute patient appointments; 6 = Close the clinic. Private sector includes 3 doctors who worked in both sectors

† Significant difference was noted between public and private sectors $(P<0.05)$ by Chi squared test 
consultation services and/or reduced consultation time. As of 4 February 2020, over 140 patients with suspected severe acute respiratory syndrome coronavirus 2 (SARS-CoV-2) infection had been encountered by 70 (14\%) surveyed family doctors; one public family doctor reported a patient who was subsequently confirmed to have SARS-CoV-2 infection. Among the surveyed family doctors, 310 (63\%) perceived needs for more training on how to deal with the COVID-19 outbreak. At time of the survey, to assist clinical decision making for diagnosing COVID-19, guidelines from the Centre for Health Protection or the Hospital Authority were used by public family doctors more frequently than by private family doctors $(n=143$ [58\%] vs $\mathrm{n}=98$ [40\%], $\mathrm{P}<0.001)$. Conversely, 195 (80\%) of the surveyed private family doctors encountered problems in stocking personal protective equipment (PPE).

\section{Changes in infection control practices in response to the COVID-19 outbreak}

Nearly all respondents wore masks during consultations $(n=490,99 \%)$ and washed hands between or before patient encounter $(n=486,99 \%)$ [Fig 2 and Supplementary Appendix 2]. A greater proportion of public than private family doctors insisted patients wear masks during consultations $(\mathrm{n}=210$ [85\%] vs $\mathrm{n}=165$ [67\%], $\mathrm{P}<0.001)$ and routinely screened patients' body temperatures $(n=211$ [86\%] vs $\mathrm{n}=183$ [75\%], $\mathrm{P}=0.002)$. In contrast, a greater proportion of private than public family doctors cleaned work surfaces with antiseptics at least once a day $(n=223$ [91\%] vs $n=200$ [81\%], $P=0.002)$ and installed air purifiers $(n=71$ [29\%] vs $n=35$ [14\%], $\mathrm{P}<0.001)$.

\section{Suggested measures to respond to the COVID-19 outbreak}

Of the respondents, 159 (32\%) answered the openended question, among which 135 (85\%) suggested measures to be instituted by government and/or local health authorities to facilitate frontline family doctors to respond to the COVID-19 outbreak (Table 2). A significant proportion of respondents $(\mathrm{n}=52,39 \%)$ appealed to the government, health service providers and/or professional bodies for securing adequate supply of PPE, especially surgical masks, for frontline healthcare workers as well as the general public. There was a strong call $(n=49,36 \%)$ for more effective public health policy to contain the outbreak, such as border control and/or quarantine measures for returning residents and travellers to reduce imported cases. Two respondents (1.5\%) had expectations for better coordination between the public and private sectors with respect to role delineation and resource allocation, for example, setting up a Primary Care Authority to maximise

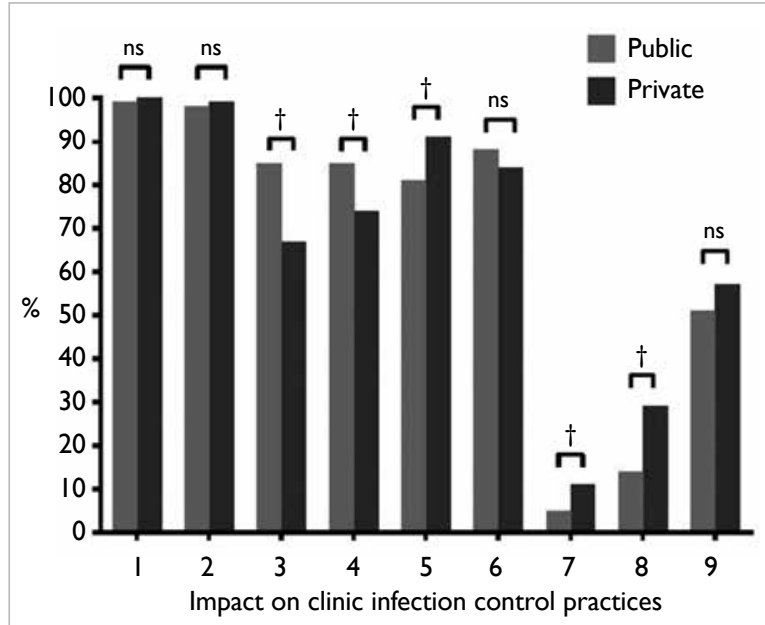

FIG 2. Clinic infection control practices of family doctors in the public and private sectors in Hong Kong in response to the COVID-19 outbreak*

Abbreviations: COVID-19 = coronavirus disease 2019; $\mathrm{ns}=$ not significant

* Impact on clinic infection control practices: I = Wear mask during consultations; $2=$ Wash hands between or before patient encounter; 3 = Insist every patient to wear a mask during consultations; 4 = Screen / measure every patient's body temperature as a routine procedure; 5 = Clean work surface with antiseptics at least once a day; 6 = All staff have to wear masks; $7=$ Open all the windows; $8=$ Install air purifiers; $9=$ Request all staff to check their body temperature before coming to work. Private sector includes 3 doctors who worked in both sectors

$\uparrow$ Significant difference was noted between public and private sectors $(P<0.05)$ by Chi squared test

efficiency and effectiveness of scattered primary healthcare delivery locally. Some family doctors $(n=9$, $7 \%$ ) advocated for the introduction of designated clinics and requested availability of rapid diagnostic tests. A few respondents $(n=8,6 \%)$ stressed the importance of public education on infection control practice and reporting accurate travel and contact history during consultation.

\section{Concerns of Hong Kong family doctors in response to the COVID-19 outbreak}

Nineteen respondents (4\%) expressed personal concerns that were consolidated into six themes (Fig 3 and online supplementary Appendix 3). The major concern was the risk of SARS-CoV-2 infection, as a result of the lack of PPE, consultation with an asymptomatic patient with SARS-CoV-2 infection, or dishonest patients with unreliable history. Owing to the lack of rapid tests and/or PPE, two private family doctors (11\%) worried that they would be unable to provide clinic services, resulting in public healthcare system overload. Three respondents (16\%) raised concerns about the need to handle and/or clarify "fake news" (ie, misinformation). 
TABLE 2. Measures suggested by surveyed family doctors to facilitate their clinic practice during the COVID-19 outbreak

\begin{tabular}{|c|c|}
\hline & $\begin{array}{c}\text { Respondents } \\
(n=135)\end{array}$ \\
\hline \multicolumn{2}{|l|}{ To the Hong Kong SAR Government, health service providers, and professional bodies } \\
\hline Monitor and/or supply personal protective equipment for healthcare workers and/or the public & $52(38.5 \%)$ \\
\hline \multicolumn{2}{|l|}{ To the Hong Kong SAR Government } \\
\hline Establish effective border control and/or quarantine measures for returning residents and travellers & $49(36.3 \%)$ \\
\hline Make accurate disclosure of travel history compulsory for patients & $2(1.5 \%)$ \\
\hline Institute penalties for dishonest patients & $3(2.2 \%)$ \\
\hline \multicolumn{2}{|l|}{ To health authorities (ie, Department of Health) } \\
\hline Provide ongoing surveillance and update of SARS-CoV-2 & $6(4.4 \%)$ \\
\hline Provide updated scientific information of SARS-CoV-2 to doctors & $6(4.4 \%)$ \\
\hline $\begin{array}{l}\text { Educate public on infection control, immunity promotion, and importance of accurate travel, } \\
\text { occupation, and contact history }\end{array}$ & $8(5.9 \%)$ \\
\hline Establish hotline for professional enquiries & $1(0.7 \%)$ \\
\hline \multicolumn{2}{|l|}{ To health service providers (ie, Hospital Authority and/or Department of Health) } \\
\hline $\begin{array}{l}\text { Establish guidelines for family doctors on appropriate triage and screening measures for handling } \\
\text { patients with suspected SARS-CoV-2 infection }\end{array}$ & $12(8.9 \%)$ \\
\hline $\begin{array}{l}\text { Establish guidelines on proper clinic protocols for infection control (eg, ventilation system, use of } \\
\text { disinfectant) }\end{array}$ & $3(2.2 \%)$ \\
\hline Arrange escort service for suspected cases to hospital & $1(0.7 \%)$ \\
\hline $\begin{array}{l}\text { Provide training for all frontline healthcare workers on proper use of personal protective equipment } \\
\text { (ie, how, when, required standards) }\end{array}$ & $9(6.7 \%)$ \\
\hline Provide rapid diagnostic tests & $5(3.7 \%)$ \\
\hline Enhance coordination of primary care service between public and private sectors & $2(1.5 \%)$ \\
\hline Establish designated clinics to handle patients with suspected SARS-CoV-2 infection & $4(3.0 \%)$ \\
\hline \multicolumn{2}{|l|}{ To other family doctors } \\
\hline Share experiences with other doctors (ie, provide peer support) & $1(0.7 \%)$ \\
\hline Research studies on COVID-19 and SARS-CoV-2 & $1(0.7 \%)$ \\
\hline
\end{tabular}

Abbreviations: COVID-19 = coronavirus disease 2019; SARS-CoV-2 = severe acute respiratory syndrome coronavirus 2

\section{Discussion}

The vast majority of surveyed family doctors were committed to discharging their duties in the early phase of the COVID-19 outbreak despite clinical uncertainties, psychological distress arising from infectious risk to self and family, and corresponding significant effects on clinical services. Only $2 \%$ of the surveyed private family doctors had closed their clinics, compared with $8 \%$ during the SARS epidemic in 2003. ${ }^{9}$ These figures were also much lower than reported absenteeism rates of healthcare workers during influenza pandemics. ${ }^{12-14}$ In the 2009 H1N1 pandemic in $2009,59 \%$ of local primary care doctors reported higher demands in clinical services. ${ }^{4}$ In contrast, in the present study, $25 \%$ of public family doctors and $45 \%$ of private family doctors reported reductions in clinical service demand. Different from an influenza outbreak when primary care doctors are tasked with providing confirmatory diagnostic tests and antiviral treatment, rapid diagnostic tests were not readily accessible in the primary care setting at time of our survey, and treatments for COVID-19 were available only in hospital settings. Patients with highly probable SARS-CoV-2 infection were sent directly to hospital isolation wards for further management. Patients suspecting themselves to have SARS-CoV-2 infection presented in large numbers to emergency departments instead of primary care clinics. Local citizens were also strongly encouraged to practise social distancing, especially avoiding areas of high contact risk, including clinics. ${ }^{15}$ Patients with other non-urgent health needs might opt to delay their clinic visits. Nevertheless, family doctors encountered probable SARS-CoV-2 infection, especially patients with milder, non-specific respiratory symptoms and with less clear travel and/or contact history. Thus, family doctors needed to remain vigilant in identifying suspected cases in the community during this period, while providing continuing care to other patients with unrelated medical conditions, mental health support for patients affected by the outbreak and educating healthy patients.

Although the mode of transmission of COVID-19 was not clearly understood in the early 


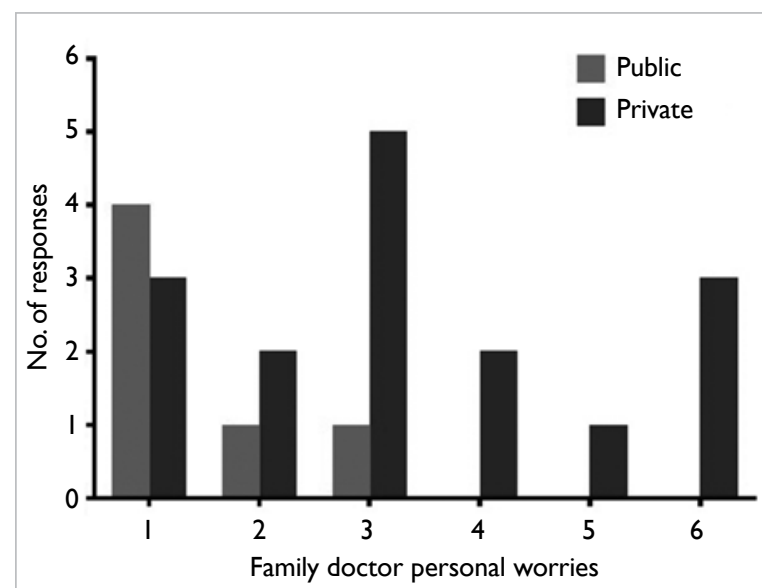

FIG 3. Concerns of Hong Kong family doctors in response to the COVID-19 outbreak*

Abbreviations: COVID-19 = coronavirus disease 2019; $\mathrm{PPE}=$ personal protective equipment

* Family doctor personal worries: I = Risks of being infected by COVID- 19 (due to the lack of PPE, asymptomatic COVID carrier and/or dishonest patients with unreliable history); 2 = Affecting family members and cross-infection; 3 = Overloading public healthcare system (due to lack of rapid tests and/or PPE); 4 = Unable to provide clinic service because of lack of PPE; 5 = Available support for family doctors infected by COVID-19;6= Need to handle/clarify fake news or misinformation

phase of outbreak, almost all surveyed family doctors readily adopted standard droplet and contact precautions, including wearing facemasks during consultation and washing hands before and/or between seeing patients as recommended by the Centre for Health Protection of Hong Kong. ${ }^{16}$ Wearing facemask during consultation became a common practice among family doctors in Hong Kong since the global outbreak of SARS.,4,9,17 Conversely, hand hygiene practices of family doctors were less consistent and varied between $45 \%$ before the H1N1 influenza epidemic ${ }^{4}$ to $70 \%$ during SARS in $2003 .{ }^{9}$ In the present survey, $99 \%$ of family doctors reported washing their hands before patient encounters during the current outbreak, which has been proven more effective than facemask wearing alone in limiting the transmission of respiratory infections. ${ }^{18,19}$ The practice of other recommended infection control measures differed between public and private family doctors, reflecting practical challenges in their implementation. A particular infection control challenge for local small-sized clinics would be the required isolation of patients with suspected SARS-CoV-2 infection, where an extra single isolation room with or without negative pressure, or even a designated isolation area $>1 \mathrm{~m}$ from the rest of the waiting area, was often unavailable. ${ }^{16}$ To protect other patients from possible cross-infection in clinics, respondents adjusted non- acute patient appointments, shortened consultation times to avoid crowding of patients in the clinic, or divert patients to other clinics. Despite the variations, these infection control measures might be contributory to the zero-infection rate observed among primary care providers in Hong Kong at the time of the survey.

Many respondents considered public health policies and interventions in response to the COVID-19 outbreak to be important. There has been an escalation of infection control responses to the COVID-19 outbreak, especially wearing of facemasks, in the healthcare ${ }^{20}$ and community settings. ${ }^{21}$ Consequently, an acute shortage of facemasks was experienced by respondents, similar to the situation observed in the US. ${ }^{22}$ Echoing the viewpoints of Australian general practitioners towards influenza pandemic management, family doctors from Hong Kong also considered that government and health authorities should be responsible for ensuring steady supply of PPE to frontline healthcare workers and/or the public.. ${ }^{23}$ A few surveyed family doctors commented that they would cease to provide clinical service if PPE became unavailable, owing to the high infection risk. Moreover, a large proportion of respondents advocated for border controls and quarantine measures to limit cross-border transmission. ${ }^{24}$ Subsequently, border controls and mandatory quarantine were implemented on people arriving from mainland China in early February $2020,{ }^{25}$ and extended to travellers from most regions around the globe in March 2020. ${ }^{26}$ These measures may have contributed to the relatively slow rise in the number of confirmed COVID-19 cases in Hong Kong.

Some private family doctors requested the introduction of designated fever clinics for the public, so that high-risk patients presenting with fever and/or respiratory symptoms could be diverted to a designated location and managed appropriately. Such arrangements would be particularly important for protecting the many small private clinics which lack the capacity to properly isolate highrisk patients. Designated clinics were successfully implemented in Hong Kong during the 2009 H1N1 pandemic $^{5}$ and in China and the US during the current COVID-19 outbreak. ${ }^{1,22}$ Unfortunately, local citizens opposed these clinics owing to a fear of COVID-19 transmission in the neighbourhood. Instead, designated doctors were assigned to attend high-risk or febrile patients in certain public primary care clinics. However, the arrangement was not clear to the public nor frontline private family doctors and symptomatic patients continued to seek care from private family doctors. Despite repeated calls for coordinated care or clear role delineation of family doctors between public and private sectors at times of outbreak, this has still not been achieved.,9 


\section{Strengths and limitations of the study}

This study had two key strengths. First, our survey was conducted in the early phase of the COVID-19 outbreak, thus the survey rapidly captured the early effects of an emerging outbreak on primary care services and reflected the clinic preparedness and needs of frontline family doctors in Hong Kong. Second, our study covered family doctors from both public and private sectors, allowing for comparison between the two sectors. Possible service gaps in the current dual-track primary healthcare system could be readily identified to inform policy makers.

A major limitation of this study was the low response rate, attributable to the relatively short survey period. Although our response rate (31\%) was lower than previous similar surveys among family doctors in Hong Kong during SARS (75\%) and H1N1 pandemic (42\%), the crude response rate was higher ( $\mathrm{n}=491$, vs 137 and 126, respectively). However, our respondents included only approximately $10 \%$ of the doctors listed in the Primary Care Directory. ${ }^{27}$ Also, only HKCFP members and fellows were targeted in this survey. Hence, the sample might not be representative of all primary care physicians in Hong Kong. Lastly, as an observational study, reporting bias existed.

\section{Conclusion}

Family doctors from both public and private sectors in Hong Kong reported willingness and preparedness to provide primary, continuous, and whole-person care to the community from the early phase of the COVID-19 outbreak. Despite limitations in clinic physical settings and potential for PPE shortages, most family doctors adopted standard precautions and effectively protected themselves and the public from cross-infection. Nevertheless, there is an obvious need for health authorities to improve role delineation and coordination between private and public primary care services and to provide relevant support during an outbreak, so that family doctors can continue to play their various roles in the community under the current dual-track primary healthcare system.

\section{Author contributions}

Concept or design: SYS Wong, EYT Yu, WLH Leung. Acquisition of data: SYS Wong, EYT Yu, WLH Leung. Analysis or interpretation of data: All authors. Drafting of the manuscript: All authors.

Critical revision of the manuscript for important intellectual content: All authors.

All authors had full access to the data, contributed to the study, approved the final version for publication, and take responsibility for its accuracy and integrity.

\section{Conflicts of interest}

All authors have disclosed no conflicts of interest.

\section{Acknowledgement}

We would like to thank The Hong Kong College of Family Physicians (HKCFP) Research and Executive Committee members who have contributed to the design of questionnaire and review of the draft, including Dr Angus Chan, Dr David Chao, Dr Catherine Chen, Dr Lap-kin Chiang, Dr Billy Chiu, Dr Cecilia Fan, Dr Ho-lim Lau, Dr Jun Liang, Dr Shuk-yun Leung, Dr Lorna Ventura Ng, Professor Martin Wong, and Dr William Wong; and Miss Erica So, Miss Crystal Yung, and Miss Angel Fung who provided administrative support for the study. We would also like to thank all the participating family doctors who responded promptly to this survey.

\section{Declaration}

This research has not been presented in any academic conference or published previously. Part of the findings from the survey was disseminated through a local press release on 10 March 2020.

\section{Funding/support}

This research received no specific grant from any funding agency in the public, commercial, or not-for-profit sectors.

\section{Ethics approval}

This study was approved by the Survey and Behavioural Research Ethics Committee of The Chinese University of Hong Kong (Ref SBRE-19-578).

\section{References}

1. Li DK, Zhu S. Contributions and challenges of general practitioners in China fighting against the novel coronavirus crisis. Fam Med Community Health 2020;8:e000361.

2. Zhong NS, Zheng BJ, Li YM, et al. Epidemiology and cause of severe acute respiratory syndrome (SARS) in Guangdong, People's Republic of China, in February, 2003. Lancet 2003;362:1353-8.

3. Tsang KW, Ho PL, Ooi GC, et al. A cluster of cases of severe acute respiratory syndrome in Hong Kong. N Engl J Med 2003;348:1977-85.

4. Wong SY, Kung K, Wong MC, et al. Primary care physicians' response to pandemic influenza in Hong Kong: a mixed quantitative and qualitative study. Int J Infect Dis 2012;16:e687-91.

5. Lee A, Chuh AA. Facing the threat of influenza pandemicroles of and implications to general practitioners. BMC Public Health 2010;10:661.

6. Lee A. Seamless health care for chronic diseases in a dual health care system: managed care and the role of family physicians. J Manag Med 1998;12:398-405.

7. Working Party on Primary Health Care. Health for all, the way ahead: Report of the Working Party on primary health care. Hong Kong: Government Printer; 1990.

8. Wun YT, Lee A, Chan KK. Morbidity pattern in private and public sectors of family medicine/general practice in a dual health care system. Hong Kong Practitioner 1998;20:3-15.

9. Wong WC, Lee A, Tsang KK, Wong SY. How did general practitioners protect themselves, their family, and staff during the SARS epidemic in Hong Kong? J Epidemiol Community Health 2004;58:180-5.

10. Wong SY, Wong W, Jaakkimainen L, Bondy S, Tsang KK, Lee A. Primary care physicians in Hong Kong and Canada-how did their practices differ during the SARS 
epidemic? Fam Pract 2005;22:361-6.

11. von Elm E, Altman DG, Egger M, Pocock SJ, Gøtzsche PC, Vandenbroucke JP; STROBE Initiative. The Strengthening the Reporting of Observational Studies in Epidemiology (STROBE) statement: guidelines for reporting observational studies. Lancet 2007;370:1453-7.

12. Balicer RD, Omer SB, Barnett DJ, Everly GS, Jr. Local public health workers' perceptions toward responding to an influenza pandemic. BMC Public Health 2006;6:99.

13. Ehrenstein BP, Hanses F, Salzberger B. Influenza pandemic and professional duty: family or patients first? A survey of hospital employees. BMC Public Health 2006;6:311.

14. Damery S, Wilson S, Draper $\mathrm{H}$, et al. Will the NHS continue to function in an influenza pandemic? A survey of healthcare workers in the West Midlands, UK. BMC Public Health 2009;9:142.

15. Fung CS, Yu EY, Guo VY, et al. Development of a Health Empowerment Programme to improve the health of working poor families: protocol for a prospective cohort study in Hong Kong. BMJ Open 2016;6:e010015.

16. Ashton LM, Hutchesson MJ, Rollo ME, Morgan PJ, Collins CE. Motivators and barriers to engaging in healthy eating and physical activity. Am J Mens Health 2017;11:33043.

17. Wong CK, Yip BH, Mercer S, et al. Effect of facemasks on empathy and relational continuity: a randomised controlled trial in primary care. BMC Fam Pract 2013;14:200.

18. Cowling BJ, Chan KH, Fang VJ, et al. Facemasks and hand hygiene to prevent influenza transmission in households: a cluster randomized trial. Ann Intern Med 2009;151:43746.
19. Aiello AE, Murray GF, Perez V, et al. Mask use, hand hygiene, and seasonal influenza-like illness among young adults: a randomized intervention trial. J Infect Dis 2010;201:491-8.

20. Cheng VC, Wong SC, Chen $\mathrm{JH}$, et al. Escalating infection control response to the rapidly evolving epidemiology of the coronavirus disease 2019 (COVID-19) due to SARSCoV-2 in Hong Kong. Infect Control Hosp Epidemiol 2020;41:493-8.

21. Leung CC, Lam TH, Cheng KK. Mass masking in the COVID-19 epidemic: people need guidance. Lancet 2020;395:945.

22. Kamerow D. Covid-19: Don't forget the impact on US family physicians. BMJ 2020;368:m1260.

23. Shaw KA, Chilcott A, Hansen E, Winzenberg T. The GP's response to pandemic influenza: a qualitative study. Fam Pract 2006;23:267-72.

24. Jefferson T, Foxlee R, Del Mar C, et al. Physical interventions to interrupt or reduce the spread of respiratory viruses: systematic review. BMJ 2008;336:77-80.

25. Viner R, Macfarlane A. Health promotion. BMJ 2005;330:527-9.

26. Magnussen CG, Koskinen J, Chen W, et al. Pediatric metabolic syndrome predicts adulthood metabolic syndrome, subclinical atherosclerosis, and type 2 diabetes mellitus but is no better than body mass index alone: the Bogalusa Heart Study and the Cardiovascular Risk in Young Finns Study. Circulation 2010;122:1604-11.

27. Hong Kong SAR Government. Primary Care Directory. Available from: https://apps.pcdirectory.gov.hk. Accessed 15 Mar 2020. 